\title{
Low Temperature Automatic Drainage Device for Water Tank of Diesel Agricultural Vehicle
}

\author{
Zhenqi Su, Yang Xu \\ Shenyang Aerospace University, Liaoning Shenyang 110136, China
}

Keywords: Automatic Drainage Device, Low Temperature, Agricultural Vehicle.

\begin{abstract}
With the mechanization increasing, the use of diesel-type agricultural vehicles is more and more common. Because of the high price of antifreeze, farmers prefer the groundwater which is easy to access, in order to prevent the freezing damage of the water tank under the low temperature environment, a single chip microcomputer is designed to control the water tank valve with low temperature and automatic drainage. Through the design of the system scheme, the temperature signal collected by the temperature sensor is transmitted to the central control system, which decides the opening and closing of the solenoid valve according to different temperature, thus realizes the effect of the low temperature automatic drainage of the agricultural vehicle tank.
\end{abstract}

\section{Introduction}

Temperature control is used in many fields, the existing methods are proportional, the integration of integral and its combination of methods, and so on to achieve the short time required for thermal balance and high temperature control accuracy of the two aspects of the implementation of a single chip microcomputer and thermal sensor combination is a good method, this method is more easily implemented and flexible and reliable.

Diesel Engine Type agricultural vehicle cooling water box antifreeze can be used antifreeze, but less than the groundwater is cheap and easy to get. In the northern, during autumn and winter, when the temperature is too low, the water in the agricultural vehicle water tank freezes, this may cause the damage to the farmers economic. Existing diesel-type agricultural vehicle water tank for manual control of drainage valves, due to work negligence, forget to operate. In order to realize the automatic protection of cooling water tank under low temperature environment, the low temperature automatic drainage device of the diesel engine water tank based on single chip microcomputer is proposed.

\section{Overall design scheme and working principle}

\subsection{Analysis system function}

In order to achieve the desired effect, it is necessary to meet the temperature precision measurement, water temperature display, sensitive movement, work alarm and work mobility.

\subsection{Design of overall structure}

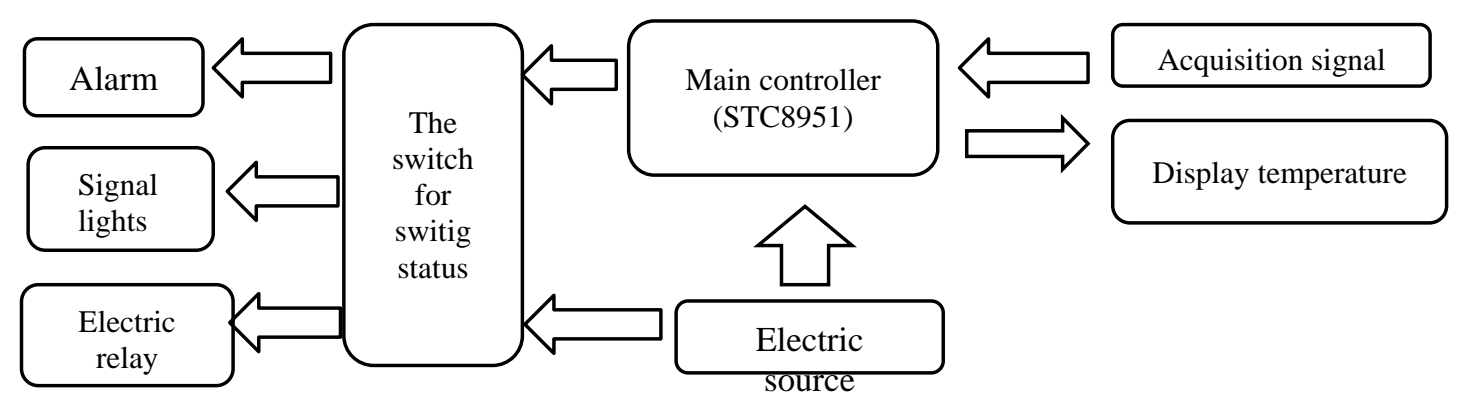

Fig.1. System structure 
The device designs the structure of signal acquisition part, temperature display part, signal processing and control part, alarm part and working part.

\subsection{Working principle of the device.}

The device uses the power supply of the locomotive, puts the temperature sensor inside the water tank to perceive the temperature signal and transmit it to the single chip computer. SCM internal procedures to deal with the temperature signal to the digital tube display water temperature, when the temperature reached 0 degrees Celsius by the Single-chip control relay work to open the valve, while the signal flashing buzzer to achieve the alarm effect. The cooling water in the water tank is discharged under the cooperation of each part. It contains the control procedure of the independent design, and the working temperature can be adjusted by program. The device is provided with a manual state switching switch, which can open the valve at any temperature and achieve the drainage effect.

\section{Hardware design}

\subsection{Collecting temperature signal.}

The use of waterproof type \$literal temperature probe, its voltage range: $3.0 \sim 5.5 \mathrm{v}$, programmable resolution of $9 \sim 12$, corresponding to the distinguished temperature of $0.5{ }^{\circ} \mathrm{C}, 0.25{ }^{\circ} \mathrm{C}, 0.125{ }^{\circ} \mathrm{C}$ and $0.0625{ }^{\circ} \mathrm{C}$, can achieve high-precision temperature measurement they are foot 1 (GND ground), foot 2 (QD data input and output pins), and foot 3 (VDD positive power).

\subsection{Main controller.}

STC89C52RC is using 8051 nuclear ISP (In System Programming) in system programmable chip, General I/O port 27/23, with EEPROM function and watchdog timer, 216 bit timer / counter. It is powered by vehicle battery through transformer.

\subsection{The part of electric relay.}

Using the constant-closed solenoid valve supply voltage $7.5-12 \mathrm{~V}$, single-chip microcomputer through the control transistor control relay, transistor overload protection.

\subsection{Alarm and signal lights.}

Alarm will sound the alarm using buzzer when the electric relay work, with light-emitting diode as the signal lights, besides, when electric relay works, the signal lights will be on.

\subsection{Temperature display.}

A total of four-digit digital tube displays the temperature values that temperature sensor upload.

\subsection{The switch for switching status.}

One side connected to the other side of the microcontroller relay, buzzer, signal, can manually control the relay to facilitate the work of different seasons in different circumstances of device mobility.

\subsection{Hardware installation model.}

The temperature sensor is placed in the cooling water of the water tank to collect the water temperature signal. Single-chip computer, monitor, buzzer, signal, state switch and other components welded on the same circuit board, external packaging Good shell fixed on the tank. Solenoid valves are installed at the bottom of the tank. Powered by car power (three-dimensional model as shown in Fig. II) 


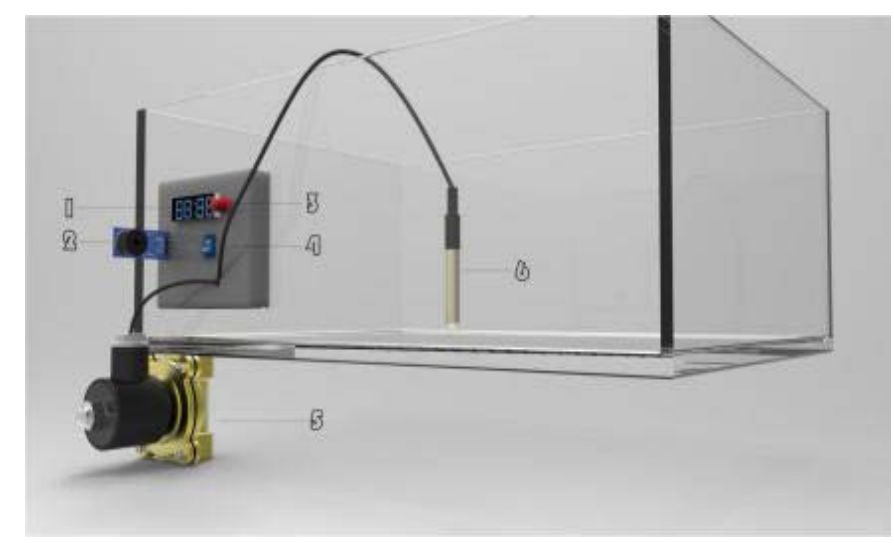

1 Nixie tube; 2 Buzzer; 3 LED; 4 The switch for switching status; 5 VVB; 6 Temperature sensor

Fig.2. Three dimensional model diagram of the device

\section{Software design}

The System program is written by the $\mathrm{C}$ language including the main function declaration global, the temperature acquisition processing part, the transmission part, the LCD display part, the scanning indication number and the control relay, the buzzer movement and so on, the program diagram just like the Figure3 shows.

The design of the system control program first declares the global pull down bus, initializes the \$literal, then writes a data from the low start to it and reads the byte every 48us from the \$literal, starting from the low. Then the temperature signal is converted and the command is sent. read a total of 16 bytes, starting from the low, and then control the digital tube (LCD) display function, first sent the selection after sending a total of four times, the digital tube display read the temperature due to the accuracy of 0.5 used rounding, the final scan display and check the Independent State switch, If the relay is closed when the indication number is not greater than 0 o'clock relays open, buzzer alarm.

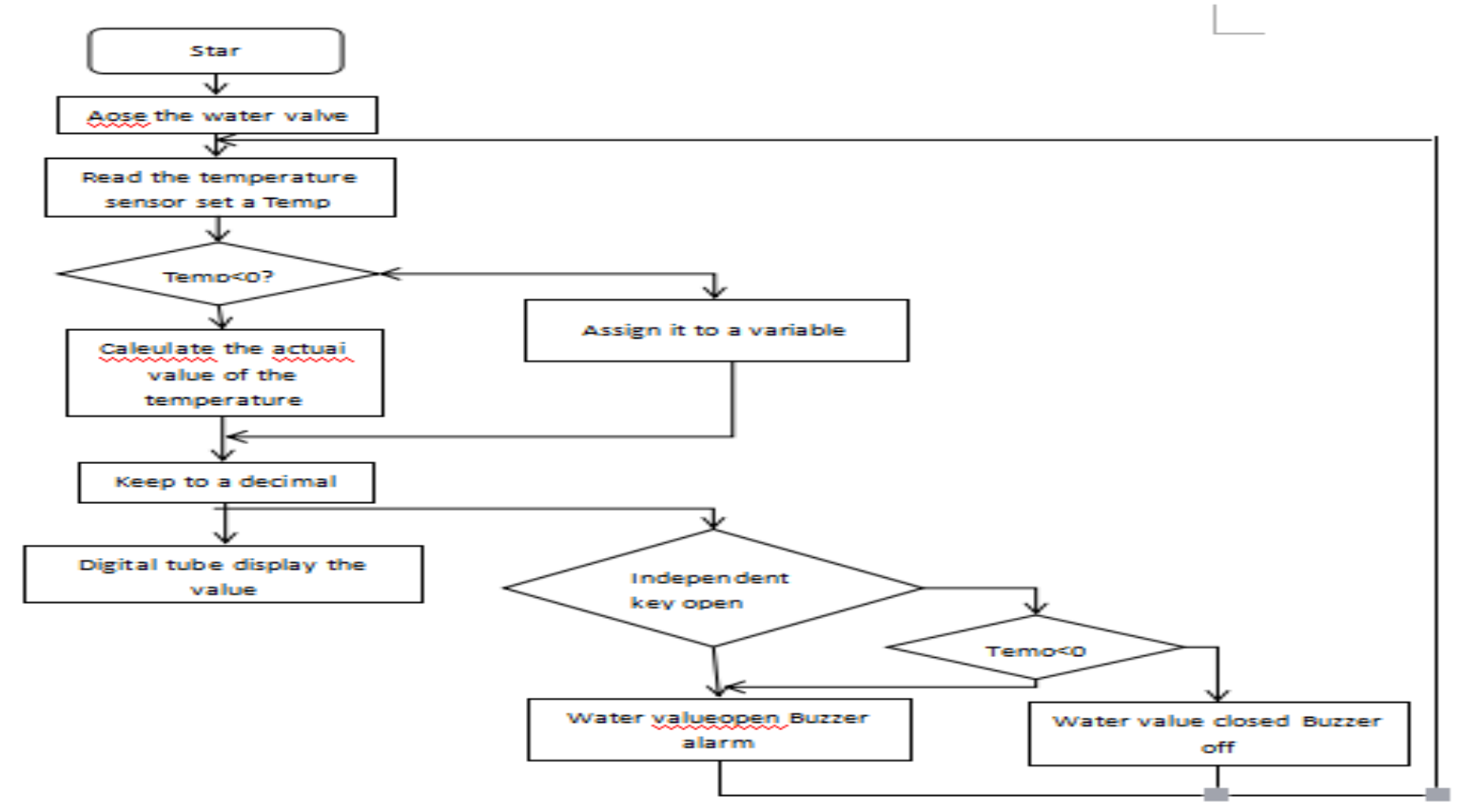

Fig.3. System program flow chart 


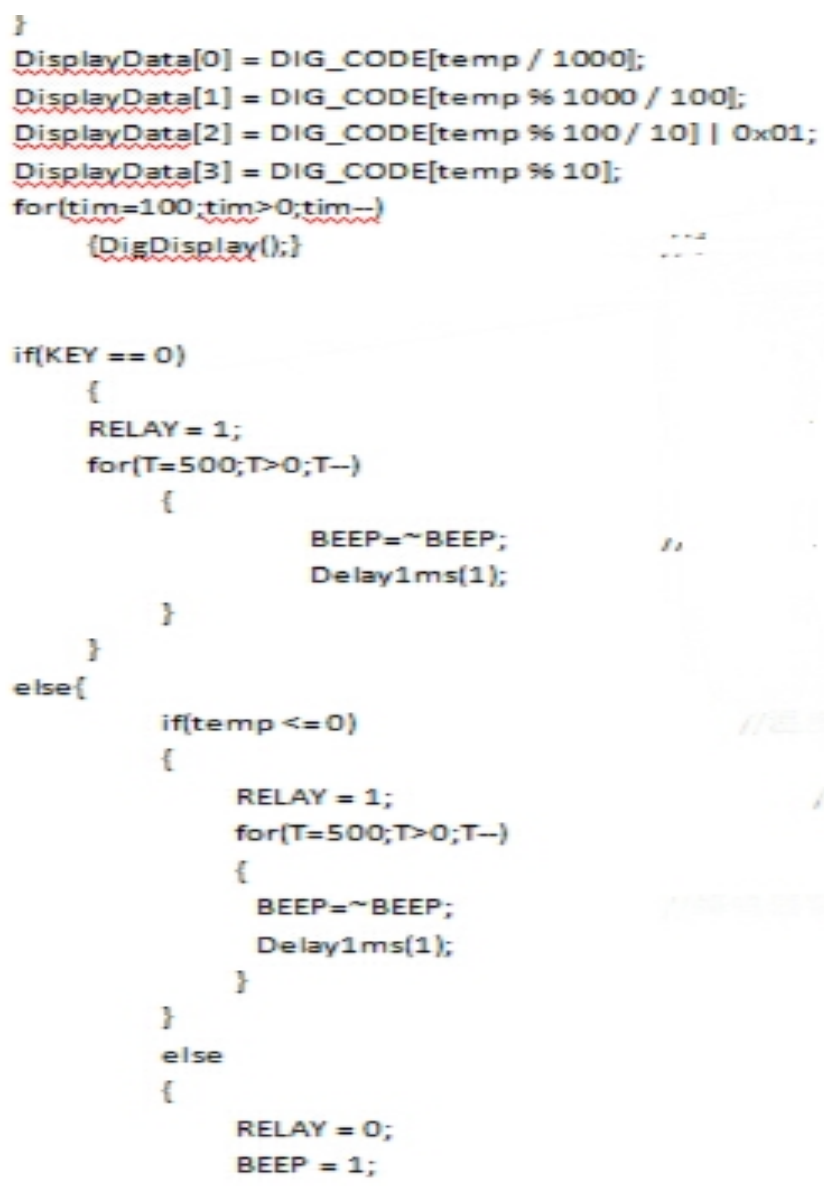

Fig.4. Main program screenshot

\section{Conclusion}

Through the design of the system and the production of the material, after many experiments, the device achieves the desired design effect.

The application of single-chip microcomputer achieves accurate measurement, drainage antifreeze, automation control, and effectively solves the problem of water tank icing. Low cost, simple structure and small size, intelligent protection effect is good, according to different needs to rewrite the program, so that the valve can work in a variety of preset temperature, has a certain value of promotion. Contains automatic, manual two modes can be switched, convenient for different seasons in different regions to play a different role, the application prospect is broad.

\section{Acknowledgements}

The Shenyang Aerospace University Student's Platform for Innovation and Entrepreneurship Training Program Foundation of China under Grant No.X1612624)

\section{References}

[1] Chen Tsuhan. Audiovisual Speech Processing. IEEE Signal Pro-cessing Magazine, 2001, 18(1): 9-21.

[2] Chen Tsuhan, Rao R R. Audio-visual Integration in Multimodal Communication. Proceedings of the IEEE, 1998, 86(5): 837-852. (In Chinese) 